\title{
COMPETENCIAS Y DESTREZAS PARA LA ACTUACIÓN PROFESIONAL EN AMBIENTES DIGITALES EN CIENCIA DE LA INFORMACION
}

\section{COMPETENCIES AND SKILLS FOR PROFESSIONAL PERFORMANCE IN DIGITAL ENVIRONMENTS IN INFORMATION SCIENCE}

\author{
José Antonio Moreiro-González' \\ Francisco Carlos Paletta ${ }^{2}$
}

\begin{abstract}
RESUMEN
Análisis de las competencias y habilidades que capacitan a los profesionales de la información para desarrollar su actividad en el entorno digital. Se ha buscado examinar el impacto de las tecnologías digitales en las tareas que ejecutan y en las estructuras del mercado laboral. Los antecedentes y el estado de la cuestión se han elaborado a partir de la recopilación y revisión de fuentes documentales con autoridad en el asunto y con oportuno interés. Asimismo, se han contrastado las consecuencias de la digitalización en el ejercicio de la profesión desde los datos obtenidos en trabajos aplicados. La exposición comienza valorando la incidencia de la tecnología en la evolución disciplinar, junto a la caracterización de los sistemas que interactúan enlazados en la red. Hasta llegar a plantear la generación y difusión abierta de los recursos y la organización y recuperación de carácter semántico. Se describen las consecuencias de la digitalización y de la gestión digital en los perfiles y requisitos laborales. Se justifica la necesidad de unas competencias específicas de carácter transdisciplinar. Alcanzando, incluso, a las consecuencias que esta situación tiene para la formación universitaria. Las conclusiones destacan la ejecución de tareas desde métodos colaborativos de asociación en la red. Se valoran las características digitales de nuestras profesiones y el tipo de profesional que busca un mercado de trabajo más complejo y transdisciplinar.
\end{abstract}

Palabras-clave: Información y Documentación. Digitalización. Gestión Digital. Competencias. Habilidades.

\section{ABSTRACT}

Analysis of the skills and abilities that enable information professionals to develop their activity in a digital environment. The aim has been to examine the impact of digital technologies on the tasks they perform and on labour market structures. The background and status of the issue have been developed from the compilation and review of authoritative documentary sources with timely interest. Likewise, the consequences of digitalization on the exercise of the profession have been contrasted from the data obtained in applied works. The exhibition begins by assessing the impact of technology on disciplinary evolution, together with the characterization of the systems that interact linked in the network. To the point of proposing the generation and open dissemination of resources and the organization and recovery of semantic character. The consequences of digitization and digital management on job profiles and requirements are described. The need for specific transdisciplinary skills is justified. We are even reaching the consequences that this situation has for university education. The conclusions highlight the execution of tasks from collaborative methods of association in the network. The digital characteristics of our professions and the type of professional looking for a more complex and transdisciplinary job market are valued.

Keywords: Library and Information Science. Digitalisation. Digital Management. Professional Tasks. Competences.

Artigo recebido em 31/03/2019 e aceito para publicação em 20/05/2019.

1 Doutor em História pela Universidade Nacional de Educação à Distância, Espanha. Professor Catedrático da Universidad Carlos III, Espanha. E-mail: jamore@bib.uc3m.es.

2 Doutor em Ciências pela Universidade de São Paulo, USP. Professor e pesquisador da Escola de Comunicações e Artes da Universidade de São Paulo, Brasil. Docente permanente do Programa de Pós-Graduação em Ciências da Informação da Universidade de São Paulo, Brasil. E-mail: fcpaletta@usp.br. 


\section{INTRODUCCIÓN}

Una de las condiciones que mejor define la vida actual es la comunicación instantánea, pues ha permitido el acceso universal al conocimiento y ha transformado y mejorado los modos de vida. Tapscott, desde una perspectiva económica, estimó las transformaciones consecuentes a la digitalización de la información y su comunicación mediante Internet (1996). Razonó su concepto de Economía digital desde las causas que habían llevado a una nueva forma de desarrollar, producir, suministrar y administrar bienes y servicios desde las aplicaciones tecnológicas. El modo renovado de comunicarnos abría el camino a una nueva sociedad que disponía del conocimiento en red, uno de los grandes hechos en el tránsito de la revolución industrial hacia la sociedad de la información. En ella, los productos que almacenan y procesan información han pasado a influir en el trabajo, en los hogares, en el entretenimiento y en la educación modificando las necesidades de información de los ciudadanos.

Si la tecnología digital permite manejar todo tipo de objetos de información y comunicarlos de manera directa y sencilla con alta calidad, no deja de ser una parte de cuantas parcelas de la vida participan cada vez más de la virtualidad. En el paso de un modelo económico al otro se fijaron unos perfiles que afectaban a todos los hechos en un alcance de servicio universal establecido desde el propio concepto interdisciplinar de Web Science (BERNERS-LEE et al., 2006). El planteamiento retomaba la red ARPANET como precedente de Internet cuando, en los años sesenta, los primeros ordenadores se intercambiaron mensajes (SHANON; WEAVER, 1981). Aunque la concepción originaria se superó pronto, abrió caminos de avance rápido que se fueron distanciando deprisa de lo establecido y abrieron una fase de vacilación. Si a lo largo de la Historia los hitos renovadores informativo-documentales tardaban siglos en producirse, ahora solo habían transcurrido unas décadas desde que la Documentación se había consolidado como ciencia. La brevedad de este lapso de ruptura estaba causada por los avances acelerados de las tecnologías electrónicas. Hasta ese momento, los medios que posibilitaban trasladar los contenidos de la información de un lugar a otro se enfrentaban a las diferentes formas de distancia y a la heterogénea materialidad de los objetos de información. Pero ahora la tecnología venía a salvar la lejanía entre la fuente de información y su consulta. De esta manera, los usuarios y los recursos entraban en un espacio en el que no hay lugares, ni distancias, ni demoras en la entrega.

En la actualidad el trabajo se transforma con mucha rapidez, tanto por influjo de la aceleración de las etapas en la vida como por la calcinación de los ciclos tecnológicos. Muchas de las ocupaciones anteriores están en trance de desaparecer y, las que no, sufren modificaciones radicales. Adaptarse 
a este cambio afecta especialmente a los inmigrantes digitales, pero alcanza también a los jóvenes, muchos de los cuales tampoco cuentan con las competencias que está pidiendo el mercado laboral.

En el cruce del comercio y la tecnología se generan ya la mayor parte de los puestos de trabajo, dentro de una transversalidad cada vez más demandada (DAVID, 2001). La incertidumbre de este cambio de época se evidencia, pues, a la hora de una inserción profesional que exige competencias digitales. Se trata de una situación nueva que apremia a una adaptación exigente, pues accedimos al oficio y a los estudios con los recursos de información bien determinados y cuando las prácticas sobre ellos y la relación con los usuarios eran tan propias que nos distinguían con claridad de otros profesionales. Muchas de ellas hoy ya no funcionan, cuando la información se genera, distribuye, gestiona y emplea en red. De nada vale que en alguna ocasión nos hayamos sentido desubicados y hayamos culpado del posible desfase al penúltimo avance tecnológico y no a la forma inapropiada de actuar ante una información que es otra en su naturaleza, comunicación y uso. Tanto profesionales como usuarios estamos afectados profundamente por un mundo en red que nos concede nuevas capacidades, pero que exige para abordarlas unas competencias y habilidades distintas.

De ahí el esfuerzo de varias generaciones de profesionales para desprenderse de prácticas que han sido adecuadas y que se han probado útiles en las anteriores condiciones, pero que ahora se han vuelto inservibles y a veces contraproducentes. Al propagarse la información digital por la red se ha ocasionado una transformación extremada en los paradigmas y en las prácticas profesionales (RUBIN, JANES, 2017) que ha originado una nueva cultura de producción y uso de la información, con consecuencias inmediatas para la adquisición de competencias y destrezas.

\section{FINES Y PLANTEAMIENTO}

Los profesionales de la Información no pueden desentenderse de esta situación. La única salida es adaptarse a las nuevas condiciones. Este tiempo de ajuste se origina en unos usuarios que no siempre están delante del mostrador o de la mesa de trabajo. Se abandonan así muchas de las anteriores actividades de intermediación, debido a la facilidad para comunicarse directamente con las fuentes de los recursos. Esto ha obligado, desde hace al menos dos décadas, a ir hacia un profesional capaz de adelantarse a las necesidades. Que no permanezca en el procesamiento y se motive más hacia la atención a los usuarios, lectores 0 , incluso, clientes si se trabaja en el sector empresarial (LEIVA, 2010). Los servicios de información buscan agilidad en la interacción con los usuarios para generales 
la mejor experiencia posible. De modo que el primer fin está en examinar si el uso de la tecnología por Ios usuarios ha provocado que los sistemas de información se muevan hacia la oferta de recursos más complejos y personalizados.

Pero la capacidad de comunicación va más allá, pues tanto la gestión de los recursos ante las dificultades económicas, como la aspiración al conocimiento abierto, se afrontan con mayor colaboración, intercambio y apoyo mutuo entre los colegas. Lo que aconseja comprobar si esa capacidad se deriva hacia la soltura en el manejo de las redes sociales y a la hora de asumir tareas educativas, como asesorar a investigadores 0 adiestrar en competencias informativas.

Si antes los diversos soportes se custodiaban en un espacio específico de tratamiento y consulta, ahora transitamos por un entorno de proyección ignota. Se deben, pues, reflejar los cambios por los que pasa la gestión de la información, con consecuencias en los centros y en su adaptación a las nuevas necesidades. Nos situamos en una intersección entre la informática, las comunicaciones y la atención a los usuarios que no es ajena a otras líneas de actividad y que nos lleva a mirar hacia dónde va la formación en Ciencia de la Información (CHAWNER, OLIVER, 2016).

Este examen no se limita a describir los precedentes y el estado de la cuestión desde las constataciones provenientes de artículos en revistas científicas, incluso revisando los libros clásicos para determinar la fundamentación teórica. Sin duda, sirven para definir los conceptos elementales a la hora de sistematizar el marco teórico conceptual y los referentes que facilitaron respaldar las ideas aquí expuestas. Cierto es que los procesos implicados en este método posibilitan la consulta de fuentes documentales relevantes para determinar las diferentes aproximaciones y valoraciones críticas sobre las consecuencias de la digitalización en la formación y en el ejercicio de la profesión. El análisis se ha completado desde otros estudios conformes con este asunto $y$, desde luego, a partir de trabajos propios que se han adentrado parcialmente en esta situación, casi todos realizados en colaboración, y que aportan el necesario argumento de los hechos, en general desde una perspectiva de investigación de carácter social. Esta preferencia se causa en su alta percepción de la vigencia de los sucesivos ciclos de innovación tecnológica para gestionar la información.

\section{¿Qué es distinto para los profesionales de la información?}

Cada vez que se produjo una revolución en la tecnología aplicada a los procesos informativos se alteraron las técnicas para gestionarlos y organizarlos. Y siempre aprovechando las ventajas que ofrecía la misma tecnología que originaba la nueva situación. ¿Cómo se controló el número de libros existentes tras la aparición de la imprenta? Con las bibliografías, es decir, con libros impresos sobre 
Ios libros impresos. ¿Y la variedad de documentos no impresos generados por la revolución industrial? Permitiendo soluciones desde los nuevos soportes, como pasó con la microfotografía. Más tarde, con las máquinas selectoras o las bases de datos, ya dentro de la profunda reforma causada por la intervención de las TIC.

Tabla 1 - Características de los ciclos evolutivos de la información

\begin{tabular}{|c|c|c|c|c|}
\hline $\begin{array}{lllllllll} & \text { e } & r & \text { i } & 0 & d & 0 & s \\
\text { esquemáticos } & & & \\
\end{array}$ & $\begin{array}{l}\text { Factores económico- } \\
\text { sociales }\end{array}$ & Rasgos y tecnología & Soportes & Sistemas \\
\hline $\begin{array}{l}\text { Antigüedad } \\
\text { Civilizaciones de } \\
\text { Oriente medio }\end{array}$ & $\begin{array}{l}\text { Bajo número de obras y } \\
\text { de posibles lectores. }\end{array}$ & $\begin{array}{l}\text { Producción de manuscritos: } \\
\text { tinta, estilos, pluma, ... }\end{array}$ & \multirow[t]{2}{*}{$\begin{array}{l}\text { Tablas de barro, } \\
\text { cerámica, madera y } \\
\text { cera. } \\
\text { Grabaciones en piedra } \\
\text { y metal. } \\
\text { Rollos de papiro, } \\
\text { pergamino ... }\end{array}$} & $\begin{array}{l}\text { Fondos en templos y } \\
\text { palacios }\end{array}$ \\
\hline Periodo helenístico & $\begin{array}{l}\text { Primeras grandes } \\
\text { colecciones, como el } \\
\text { Museo de Alejandría. }\end{array}$ & & & $\begin{array}{l}\text { Técnicas pre- } \\
\text { bibliotecarias: } \\
\text { Syllabus: identificación } \\
\text { Pinakes: ordenación } \\
\text { Canon: repertorios }\end{array}$ \\
\hline Edad Media & Misión de custodia & Copia de códices. & Códices & Bibliotecas monásticas \\
\hline Desde el S. XIII & $\begin{array}{l}\text { Mayor número de } \\
\text { lectores }\end{array}$ & $\begin{array}{l}\text { Incremento en la circulación } \\
\text { de ejemplares }\end{array}$ & $\begin{array}{l}\text { Becerros, papel. } \\
\text { Impresión mecánica por } \\
\text { bloques }\end{array}$ & $\begin{array}{l}\text { Bibliotecas de las } \\
\text { universidades }\end{array}$ \\
\hline Edad Moderna & $\begin{array}{l}\text { Imprenta- Bibliografías: } \\
\text { racionalismo en la } \\
\text { producción y el control } \\
\text { de la información }\end{array}$ & $\begin{array}{l}\text { Abundancia de libros; } \\
\text { Adaptación a los lectores; } \\
\text { Estandarización de los } \\
\text { registros (Maunsell) }\end{array}$ & $\begin{array}{l}\text { Impresión por tipos } \\
\text { (Gutenberg) } \\
\text { Libros. Copias ilimitadas } \\
\text { de cada original }\end{array}$ & $\begin{array}{l}\text { Listas de existencias } \\
\text { comerciales o } \\
\text { académicas; } \\
\text { Intermediación } \\
\text { bibliográfica; } \\
\text { Identificación por } \\
\text { autores y registros }\end{array}$ \\
\hline Siglo XVII & $\begin{array}{l}\text { Revolución científica. } \\
\text { Ciencia moderna }\end{array}$ & $\begin{array}{l}\text { Comunicación periódica } \\
\text { del conocimiento y de la } \\
\text { información científica }\end{array}$ & Revistas & $\begin{array}{l}\text { Revistas con } \\
\text { referencias a los } \\
\text { materiales primarios } \\
\text { (S. XVIII) }\end{array}$ \\
\hline
\end{tabular}




\begin{tabular}{|c|c|c|c|c|}
\hline Siglo XIX & Big Science (Price) & $\begin{array}{l}\text { Gran número e intensa } \\
\text { difusión de las revistas. } \\
\text { Interés por las publicaciones } \\
\text { vivas }\end{array}$ & Revistas & $\begin{array}{l}\text { Hacia la } \\
\text { Documentación }\end{array}$ \\
\hline Siglos XIX-XX & $\begin{array}{l}\text { Documentación (Otlet, La } \\
\text { Fontaine, Dewey) }\end{array}$ & $\begin{array}{l}\text { Internacionalización de la } \\
\text { información: IIB-FID; } \\
\text { Superación de los impresos } \\
\text { como soporte único }\end{array}$ & $\begin{array}{l}\text { Documentos } \\
\text { producidos por } \\
\text { medios técnicos de la } \\
\text { revolución industrial: } \\
\text { fotografía, cine, radio, } \\
\text { discos, informes } \\
\text { científicos, ... }\end{array}$ & $\begin{array}{l}\text { Normalización de los } \\
\text { procesos Clasificación } \\
\text { de las colecciones } \\
\text { (DDC-UDC) } \\
\text { Profesión con } \\
\text { formación universitaria }\end{array}$ \\
\hline Postguerra mundial II & Informe Weinberg & $\begin{array}{l}\text { Reproducción documental; } \\
\text { Automatización de la } \\
\text { recuperación; } \\
\text { Teoría matemática de la } \\
\text { Comunicación }\end{array}$ & $\begin{array}{l}\text { Técnicas } \\
\text { preinformáticas: } \\
\text { Microcopias; microfilm; } \\
\text { selectores fotográficos; } \\
\text { Fichas perforadas }\end{array}$ & $\begin{array}{l}\text { Recuperación de la } \\
\text { Información }\end{array}$ \\
\hline Años 60-70 & $\begin{array}{l}\text { Ciencia de la Información } \\
\text { Informatika }\end{array}$ & $\begin{array}{l}\text { Tecnología informática; } \\
\text { Lenguajes coordinados para } \\
\text { la recuperación; Análisis } \\
\text { bibliométrico; Comunicación } \\
\text { de la información }\end{array}$ & $\begin{array}{l}\text { Formatos electrónicos } \\
\text { de datos }\end{array}$ & $\begin{array}{l}\text { Gestión documental } \\
\text { empresarial } \\
\text { Recursos sometidos a } \\
\text { las leyes del mercado }\end{array}$ \\
\hline S. XX. Décadas finales & Internet & $\begin{array}{l}\text { TIC; Estrategia empresarial; } \\
\text { Economía colaborativa; } \\
\text { Representación e } \\
\text { interoperabilidad } \\
\text { semánticas; Redes de } \\
\text { contenidos hipertextuales; } \\
\text { Usuarios digitales }\end{array}$ & $\begin{array}{l}\text { Documentos digitales } \\
\text { Digitalización }\end{array}$ & $\begin{array}{l}\text { Almacenamiento y } \\
\text { procesamiento de } \\
\text { datos multimedia } \\
\text { enlazados; } \\
\text { Disponibilidad } \\
\text { universal de acceso } \\
\text { y comunicación; } \\
\text { Conocimiento abierto }\end{array}$ \\
\hline Década de 2010 & & & & $\begin{array}{l}\text { Rapidez en el proceso } \\
\text { de transformación } \\
\text { digital }\end{array}$ \\
\hline
\end{tabular}

Fuente: MOREIR0, 1990. Elaboración propia. 
Los servicios de información están ahora enlazados. Este atributo facilita la cooperación con otras unidades, con usuarios y colegas conectados desde cualquier parte del mundo y con los centros de estudio, investigación y negocio. Lo que integra a las unidades de información en el sector tecnológico, entre los servicios más innovadores, a la vez que las obliga a ser ágiles y flexibles (TAM, ROBERTSON, 2002). La interactividad intrínseca de lo digital facilita la participación de cuantos intervienen en los actos comunicativos. No solo de las fuentes y los gestores, sino de los usuarios, pues la brecha entre ellos y las fuentes está desapareciendo al poder accederlas de forma directa y fácil. No hay duda sobre las consecuencias que ha tenido la interacción de los profesionales con la información, las TIC y los sistemas de información. Su manera de actuar, hasta ahora centrada en canalizar y mediatizar los procesos conducentes al acceso y entendimiento de la información, ha cambiado. Lo digital ha emergido fuera de los cauces de organización precedentes. Pero, preocupa más la desigualdad generada por los cambios tecnológicos y económicos al dividir las personas entre las que pueden acceder a las nuevas infraestructuras y servicios y quienes no pueden, con lo que se exponen a quedarse sin participar en la vida económica y social y, desde luego, en la igualdad por el acceso al empleo (BRYNJOLFSSON; MCAFEE, 2011).

No fue menos importante el tele-acceso desde un ordenador a información alojada en bases distantes. En los años setenta la comercialización de las Bases de Datos en línea DIALOG y BRS permitió el acceso virtual a la información de las empresas, universidades y centros de investigación e, incluso, de los usuarios personales. El desarrollo inicial de las TIC formalizó la vieja aspiración de Otlet al acceso universal (MOREIRO, 1990). Lo corroboró, después, Internet al eliminar la mayoría de los impedimentos físicos para acceder a la información científica. Este hecho tuvo efectos notorios en el sistema de comunicación académica, como demuestra la manera en que se indizan los documentos académicos, así Google Scholar, o en situarse como base del movimiento "Open Science" para compartir todos los productos científicos (Open access, Open data, Open research, Citizen Science, ...). Con incidencia especial en la responsabilidad de los profesionales a la hora de suministrar recursos a los investigadores y estudiantes, tanto los facilitados por las bibliotecas, como los datos generados en los laboratorios e institutos de investigación (LYON, 2016).

\section{EFECTOS DE LA DIGITALIZACIÓN EN EL MERCADO DE TRABAJO}

Con Internet se han estrenado muchas profesiones y otras, ya existentes, se han tenido que actualizar tras pasar a efectuar su trabajo en línea. Entre ellas se sitúan, en participación oportuna, 
los perfiles de Ciencia de la información. No hay duda de que una de las palancas de la estrategia digital se apoya sobre la información digital, junto a la automatización de los procesos mediante IA, la conectividad y el acceso de los usuarios a Internet. Por otra parte, la inmediatez de uso es crítica porque Ios usuarios quieren información en tiempo real. Los usuarios pueden aparecer en cualquier lugar y usando cualquier canal, pues se valen de las redes sociales y las aplicaciones móviles. De forma que la circulación de la información se ha vuelto electrónica. Esta situación era hace poco desconocida y Ios servicios de información han aprendido a enfocarla. Aparecen, pues, nuevos modelos de servicio $y$, en consecuencia, nuevos puestos de trabajo a la vez que se elimina mucha burocracia y las tareas repetitivas mediante la automatización de los procesos técnicos. La llave del éxito proviene de unas competencias que son comunes a diferentes sectores de actividad con una importancia decisiva para su desarrollo, como la capacidad para gestionar grandes cantidades de información, la orientación a Ios usuarios, el control de las herramientas informáticas y de su entorno de aplicación. Por más que en algunos de los procesos resulte difícil definir la frontera entre lo tradicional y lo digital.

Aun así, los elementos de esta enumeración obligan a especializarse en las herramientas y habilidades de los ambientes digitales y a hacerlo en necesaria formación permanente, más allá de la recibida en la universidad. Además de encontrarnos en un sector en cambio constante en medio de una sociedad muy diferente, la recesión económico-financiera complicó aún más un ámbito laboral que arrastraba, desde hace al menos dos décadas, problemas de identidad, con la disminución del empleo en el sector público y el progresivo aumento del procedente del sector privado (TEJADA et al., 2014). Junto a problemas de reconocimiento social y salarial que demandan una serie de competencias y salidas profesionales más amplias, por lo que está obligado a buscar nuevas oportunidades (ORTIZ et al, 2013). Sin embargo, la misma recesión impulsó una mayor cooperación entre los profesionales de la información. Era inevitable que se comunicasen entre ellos y saber hacerlo a través de las redes sociales.

Si hubo reticencias iniciales hacia las TIC desaparecieron pronto, al mostrarse su efectividad para aumentar la proyección y el alcance de los servicios ofertados y, en consecuencia, mejorar la satisfacción de los usuarios. En poco tiempo, el uso de Internet se hizo general para acceder a todo tipo de registros digitales. Los profesionales de la información no podían renunciar a facilitar el acceso a los recursos y el suministro de servicios mediante la red de forma más rápida y fácil. De una dificultad volvió a salir una solución, sin evitar la consiguiente modificación de los procesos técnicos y de mentalidad laboral. Internet forzó a una postura abierta. Si los usuarios encontraban su propia información no 
precisaban acudir al documentalista, por lo que este, en justificación de su existencia, estaba obligado a salir hacia los usuarios. Se trataba de demostrarles que se podía mejorar su experiencia informativa.

Desde los años de crisis económica, las ofertas de trabajo fueron dejando de limitar los puestos a una sola titulación (Ciencia de la Información) o a un solo perfil, en general de servicio público, como archivero, bibliotecario, documentalista, ... Y pasaron a remitirse a perfiles mixtos, a los que podían concurrir titulados en distintos sectores, en especial en Gestión empresarial, Márketing, Computación o Comunicación, siempre desde la perspectiva de los procesos digitales (DE LIMA, 2007). La presencia en equipos transdisciplinares no solo afectó a nuestro ámbito, pues se extendió a todos los campos de actividad empresarial, institucional e incluso a los organismos oficiales, en una coyuntura que ha ofrecido nuevas salidas comunes y que, por tanto, también ha abierto el abanico de perfiles a los que pueden optar nuestros titulados (TEJADA et al., 2014).

Lo digital se atiende, pues, desde perfiles laborales de carácter transversal. Esta nueva realidad se demuestra en la solicitud de profesionales con competencias y habilidades digitales que puedan transferirse a múltiples sectores. Nos referimos a profesionales que, más allá de trabajar en unidades de información autónomas, se encuadran en servicios de información de toda clase de organizaciones con presencia digital en sus documentos, procedimientos y gestión. Actuando, por tanto, en cualquier empresa y sector de actividad. A estos efectos, son muy representativos los resultados obtenidos por la profesora Bahia (2016) en su análisis de anuncios de empleo en la web, que enfatiza la presencia de titulados en sistemas de información de estos ámbitos:

Tabla 2 - Sectores de gestión y tratamiento digital de documentos.

\begin{tabular}{|l|l|}
\hline Sector & $N^{0}$ de anuncios \\
\hline Servicios hospitalarios y médicos & 2990 \\
\hline Despachos de abogados y asesorías & 2102 \\
jurídicas & \\
\hline Documentación jurídica & 541 \\
\hline Servicios de recursos humanos & 423 \\
\hline Asesorías de administración de empresas & 110 \\
\hline Estudios de arquitectura & 80 \\
\hline Gestorías de cobros & 70 \\
\hline Asesorías de contabilidad & 64 \\
\hline Estudios de Ingeniería civil & 22 \\
\hline
\end{tabular}

Fuente: (BAHIA, 2016). Elaboración propia. 
Se destaca la presencia de los sistemas de información médica y legal, dos campos de tradicional actividad documental, pero que ahora la han potenciado favorecidos por la digitalización de los procesos. En el número de ocurrencias se incluyen los múltiples niveles de entradas específicas correspondientes a cada uno de los sectores identificados, como por ejemplo los prontuarios y protocolos médicos que están recalculados dentro de la Gestión de información en servicios médicos.

De forma que los perfiles relacionados con los procesos digitales no se solicitan solo en ofertas destinadas a profesionales de Ciencia de la información, sino también en las de sectores de actuación cercanos, como los medios de comunicación, los centros educativos, los servicios jurídicos y de consultoría o los servicios informáticos. No olvidemos que nuestra actuación fue siempre interdisciplinar: en una biblioteca escolar hay que contar con competencias en Educación; en una biblioteca universitaria habrán de ser en una especialidad concreta; para un archivo histórico es inevitable contar con destrezas de Paleografía y Diplomática; lo mismo que en un museo donde se necesitarán destrezas sectoriales acordes con los aspectos cognitivos, culturales o sociales implicados. A este listado se añaden otros ramos promovidos por la transversalidad de los contenidos y la gestión digitales. Situándonos en medio de un mestizaje de áreas tanto respecto a la formación de proveniencia como en los numerosos sectores de aplicación profesional (INESDI, 2015):

- $\quad$ Dentro del área de Estrategia y Gestión de Negocio (FTI-AMETIC, 2012) se sitúan los de Especialista en SEO/Analítica web, Responsable de estrategia digital, Experto en proyectos digitales, Analista de información digital, Consultor de arquitectura de la información/usabilidad, Especialista en experiencia de los usuarios o Gestor de proyectos web.

- Que se relacionan asimismo con los perfiles combinados de Comunicación y Gestión de contenidos digitales, como los de Gestor de comunidades, Especialista en márketing digital, Editor de contenidos, Experto en comunicación digital o Especialista en medios de comunicación de las redes

Todas estas denominaciones y sus posibles variantes se han convertido en salidas comunes para los profesionales de la información con competencias digitales (ESPAÑA, 2015). Se trata de perfiles que acentúan su estrecha cercanía con las TIC y la comunicación con los usuarios. Este hecho no resta oportunidades laborales a aquellos, al contrario, las aumentan, pues persisten los perfiles precedentes, aunque estén modificados por la transformación digital. 
Cierto es que las áreas funcionales antes consideradas, como el márketing (incluso en Facebook existe un grupo de Marketing Digital para Instituições Educacionais), la comunicación, la programación, el diseño visual e, incluso, las relacionadas con la creatividad artística han sido siempre colindantes a las funciones atendidas por los profesionales de la información, que no resultan factibles lejos de la transformación digital de los propios sistemas de información y, desde luego, de la intervención en empresas y organizaciones ajenas donde aquellas se requieren. Esta situación nos convierte en un sector incorporado al gran grupo de las TIC, aunque sin abandonar las tareas y los procedimientos técnicos propios. Pero, cada vez más, con un enfoque similar al seguido por los depósitos de datos en las empresas. Lo que se refleja de inmediato en la producción, el tratamiento y el consumo de información:

- $\quad$ Pues el soporte digital ha favorecido el procesamiento técnico común de los anteriores soportes diversificados. Lo que acerca cada vez más las funciones y los procesos seguidos en los diferentes sistemas documentales.

- $\quad$ La propia red facilita la comunicación de documentos, procesos y tareas. En especial con el crecimiento de los documentos multimedia y los contenidos sociales, tal como se destaca en los Subsectores de la Industria de contenidos digitales (EUROPEAN COMMISSION, 2006). Donde, asimismo, se reflejan subsectores ligados al campo documental como el de las publicaciones (periódicos, revistas y libros).

- $\quad$ Al tiempo que se determinan otros sectores de aplicación cuya importancia proviene de los abundantes contenidos que generan en Internet, como el comercio, la salud (de gran relieve en el informe FTI-AMETIC de 2012), el turismo, la educación, el gobierno abierto, la administración de empresas y la contabilidad.

- E incluso la capacidad para identificar, definir y planificar la adopción de tecnologías y aplicaciones Big Data para la gestión de datos e información en los procesos documentales, en especial Ios académicos. Igual que en empresas y en proyectos de carácter industrial, donde pueden actuar, como controladores de documentos, profesionales TIC, documentalistas, gestores de proyectos y consultores que, como los anteriores, se mueven con una visión más amplia a la puramente informativo-documental (SAYÃO, SALES, 2012).

De modo, que los profesionales de la Información deben de estar cualificados para actuar en diferentes subsectores de la economía digital, tanto en perfiles reconvertidos como en otros de 
reciente creación. A atenderlos no van ellos solos. Sino que compiten con formados en titulaciones de carácter transversal, lo que suele generar confusión respecto a los empleos a los que debe aspirar un profesional de la información. Ya que muchos de los puestos, especialmente en el sector privado, no fijan diferencias con los profesionales de otros sectores que suelen provenir de las titulaciones de Comunicación, Humanidades, Administración y Gestión o Informática. Ante lo que puede aflorar un cierto sentimiento de privación de funciones que se consideran propias. Pero nuestra mirada tiene que ser más amplia, pues las empresas e instituciones eligen a quienes ven más capacitados para atender sus necesidades.

\section{HABILIDADES DIGITALES Y EJERCICIO DE LA PROFESIÓN}

La irrupción de los recursos electrónicos y su transmisión por Internet fueron un revulsivo para los procesos tradicionales. Si se comparan, en ligero apunte, las prácticas actuales de catalogación e identificación de los documentos con las de hace veinte años, se percibe que incluso los propios conceptos de catalogación y de identificación se han renovado para atender al número inmenso de nuevos documentos y a sus tan variados soportes. Pero más se ha transformado el proceso técnico. Primero, por la automatización de los registros con el formato MARC y, luego, al establecerse Ios OPAC. Más tarde, los catálogos en red se hicieron cooperativos mediante el protocolo Z39.50, con lo que se evitaba tener que repetir los registros (MODESTO, 2007). Hasta llegar a la catalogación de los recursos electrónicos con metadatos que, como Dublin core, incluso se alejan de lo fijado en las reglas de catalogación. Todo ello facilitó que la propia red haya pasado a catalogar e indizar los documentos que contiene como bien demuestra el buscador Google (PARK et al. 2009).

Otro tanto sucede con la atención a los usuarios. Quien más visita una biblioteca 0 un archivo no tiene por qué acudir en persona para consultar sus fondos. Se puede ser un usuario pertinaz y no acudir en meses a sus locales. Por más que mantengan su vigencia los servicios de nuestro sector, siempre modificados por las condiciones digitales. Desde proporcionar acceso a las necesidades de telecomunicación de los usuarios hasta suministrarles conocimientos, habilidades y desarrollo personal en el uso eficaz de la información (ALA, 2016). Si bien la difusión digital ha hecho que ni siquiera sean suyos la mayor parte de los documentos que distribuyen los centros, pues proceden de intercambio 0 de colecciones en línea (ROSS, SENNYEV, 2008). 
Los hábitos de información van cambiando a medida que Internet ofrecen más opciones. De modo que no resulta extraño que, al analizar los perfiles profesionales demandados en las ofertas de empleo, Tejada, Chacón y Moreiro (2014) encontrasen que un porcentaje significativo de las contrataciones se relacionasen con el sector digital, sin contar las que se podían incluir en ofertas más genéricas:

- $\quad$ Procesos de digitalización y asignación de metadatos (3 \%).

- $\quad$ Nuevos perfiles digitales (3\%).

- $\quad$ Especialista en digitalización (2\%).

- $\quad$ Gestión de contenidos digitales (0,9\%). Y aún:

- Encargado de archivo digital $(0,16 \%)$.

○ Encargado de la preservación digital $(0,16 \%)$.

- Catalogador de contenidos digitales $(0,16 \%)$.

Sin duda, la función en el servicio público sigue siendo un atractivo considerable a la hora de cursar estudios universitarios en las especialidades de Ciencia de la información. Algunos de los procesos de ingreso aún mantienen el habitual carácter teórico, sin plantearse ejercicios prácticos o sin abordar las actuales bases del área. Sin embargo, la apertura hacia asuntos y problemas profesionales actuales se muestra cada vez con mayor frecuencia en las competencias exigidas a los candidatos. Hasta, con relativa frecuencia, se dejan ver enfoques últimos, sobre todo en Ilamamientos para puestos directivos. Entre las más actualizadas, suelen requerirse estas competencias:

- $\quad$ Conocimiento de la interoperabilidad de los SOC.

- $\quad$ Conocimiento avanzado de procesos de digitalización y de edición electrónica.

- $\quad$ Gestión de recursos electrónicos mediante sistemas integrados.

- $\quad$ Empleo de herramientas de búsqueda electrónica.

- $\quad$ Envejecimiento de los sistemas y formatos electrónicos.

- $\quad$ Evaluación y puesta en marcha de sistemas de conservación de los documentos electrónicos.

- $\quad$ Experiencia con los requisitos de la web semántica.

- $\quad$ Asignación de metadatos enlazados en los documentos digitalizados.

- $\quad$ Manejo de herramientas para el análisis de datos.

- $\quad$ Producción normalizada de documentos electrónicos. 
Esta relación podría extenderse a todo cuanto afecta a las necesidades del personal y a las herramientas requeridas en colecciones, operaciones y aplicaciones de tecnología digital en servicios de información (CHOI, RASMUSSEN, 2009). Es evidente que las TIC fortalecen y aumentan la posibilidad de comunicación, participación y contribución virtual en el intercambio de ideas con los usuarios. Sin duda, motivan y condicionan los conocimientos necesarios para actuar con flexibilidad y adaptación a los cambios permanentes en la sociedad y en las exigencias laborales (CASTELLS, 2008).

Tabla 3 - Conocimientos y tareas más solicitados

\begin{tabular}{|l|c|}
\hline Conocimientos y tareas & Rango \\
\hline Gestión de contenidos Web & 1 \\
\hline Gestión y posicionamiento SEO & 1 \\
\hline Atención a los usuarios & 2 \\
\hline Comunicación e imagen corporativa & 2 \\
\hline Digitalización & 2 \\
\hline Documentación digital & 2 \\
\hline Edición y diseño gráfico & 2 \\
\hline Tratamiento de la imagen digital & 2 \\
\hline
\end{tabular}

Fuente: (MOREIRO et al., 2009). Elaboración propia.

Un buen ejemplo de la rápida evolución de los requisitos digitales para la actuación profesional lo marca el hecho de que en el año 2010 los procesos de digitalización de colecciones documentales eran una de las actividades más solicitadas en las ofertas de empleo (VERGUEIRO, MOREIR0, 2015). Mientras que, menos de siete años después, ya se pedía experiencia en digitalización, con habilidades de cara a la evaluación de los soportes digitales, su procesamiento y organización e, incluso, su análisis e interpretación. Hecho que refleja el desplazamiento hacia unos procesos y una gestión más variados, especializados y complejos (REYES et al, 2017).

Es evidente la progresión en la demanda de competencias y habilidades digitales cada vez más complejas y variadas. Su relevancia y ponderación quedaron bien establecidas en la investigación doctoral realizada por Eliana Bahia (2016). 
Tabla 4 - Competencias y habilidades digitales solicitadas.

\begin{tabular}{|c|c|c|c|}
\hline $\begin{array}{l}\text { Competencias y } \\
\text { habilidades }\end{array}$ & \begin{tabular}{|l|}
$N^{o} \quad$ de \\
ocurrencias
\end{tabular} & $\begin{array}{l}\text { Competencias y habilidades en } \\
\text { análisis y representación de la } \\
\text { información }\end{array}$ & $\begin{array}{l}\mathrm{N}^{0} \text { de } \\
\text { ocurrencias }\end{array}$ \\
\hline $\begin{array}{l}\text { Procesamiento técnico y } \\
\text { supervisión de la digitalización }\end{array}$ & 611 & Descripción de documentos digitalizados & 321 \\
\hline $\begin{array}{l}\text { Manejo de documentos } \\
\text { electrónicos }\end{array}$ & 599 & $\begin{array}{l}\text { Análisis y representación de la información } \\
\text { digital }\end{array}$ & 250 \\
\hline Cultura en diseño web & 310 & Indización de imágenes & 213 \\
\hline Aplicación de tecnología Web & \multirow[t]{7}{*}{$<300$} & Recuperación de documentos digitales & 114 \\
\hline $\begin{array}{l}\text { Conocimientos en Informática } \\
\text { e Internet }\end{array}$ & & Indización de documentos digitales & 69 \\
\hline $\begin{array}{l}\text { Conocimientos de archivo } \\
\text { digital }\end{array}$ & & Clasificación de documentos digitales & 23 \\
\hline $\begin{array}{l}\text { Conocimiento de márketing } \\
\text { digital }\end{array}$ & & & \\
\hline $\begin{array}{l}\text { Organización de los flujos de } \\
\text { información }\end{array}$ & & & \\
\hline $\begin{array}{l}\text { Gestión y seguridad de } \\
\text { documentos electrónicos }\end{array}$ & & & \\
\hline Creación de archivos digitales & & & \\
\hline Cultura en digitalización & \multirow[t]{3}{*}{$<200$} & & \\
\hline Digitalización & & & \\
\hline Legislación sobre digitalización & & & \\
\hline
\end{tabular}

Fuente: (BAHIA, 2016). Elaboración propia.

\section{PERFILES DIGITALES Y FORMACIÓN PARA LA PROFESIÓN}

Conforme se van perdiendo algunas de las tareas y perfiles relacionados con los espacios físicos tradicionales: bibliotecas, archivos, servicios de documentación, se progresa en actuaciones de carácter digital. Este hecho supone que en gran parte de sus actividades ni siquiera permanezcan los nombres anteriores, ni para referirse a los técnicos, ni a sus ocupaciones, ahora enmarcadas por la cercanía con:

- $\quad$ Arquitectura y modelado de la información, posicionamiento.

- Marketing, asesoría y consultoría de la información.

- Comunicación en red.

- Vocabularios semánticos y representación del contenido. 
- $\quad$ Estadística para el procesamiento de los datos.

- $\quad$ Ética en el uso y en los valores.

- $\quad$ Derechos sobre el empleo de los datos y de la información.

Las TIC modifican de continuo el enfoque de los servicios de información. Van más deprisa que el tiempo disponible para formarse. Lo transitorio se ha vuelto permanente y sitúa a los profesionales, desde el enfoque diacrónico, en un cambio radical y, desde el sincrónico, en un desasosiego continuado respecto a la respuesta que pueden dar a las exigencias que les plantean sus servicios y unidades. Sin olvidar que las organizaciones tienen mucha documentación en formatos físicos susceptible de convertirse a un formato digital para favorecer su gestión y difusión electrónicas. Estos obstáculos se suman al desafío de diseñar y organizar el trabajo a partir de las destrezas que deben poseer los nuevos empleados y de la redistribución de tareas. Sin desentenderse de ayudar a los veteranos a obtener esas destrezas para acoplarse a unas actividades y servicios tan renovados.

Todas estas evidencias permiten afirmar que los perfiles técnicos de índole digital han pasado a tener un marcado protagonismo en los objetos de trabajo, las labores, las normas y los procesos atendidos por los profesionales. La mayor complejidad de este escenario hace que los límites precedentes de actuación disciplinar vayan desapareciendo ante problemas cuyo estudio solamente es abordable desde enfoques compuestos y en el aprecio por los espacios de convergencia. No se trata de un planteamiento derivado de la reflexión, si no consecuencia de la irrupción de una tecnología que ha cambiado las reglas, como ya había sucedido en momentos precedentes. Ahora vivimos en una realidad enlazada cuyo análisis conceptual y práctica diaria tiene que hacerse desde la interpretación de su complejidad. No podemos alcanzar una valoración epistemológica de la información enlazada lejos de la reagrupación cognitiva que este hecho supone. Y si la naturaleza de la información se ha vuelto transdisciplinar, sus aplicaciones y su estudio también. ¿Cuánto falta para que los centros de información cuenten con asistentes virtuales para el trato directo con los usuarios? En estas circunstancias, no hay suficientes personas matriculadas en las carreras que relacionan las TIC con la gestión de la información

Además, los centros, instituciones y empresas tienen que comprometerse a apoyar a sus profesionales y a facilitarles la actualización formativa que les permita contar con las habilidades necesarias para seguir en la onda. La actualización es fundamental para atender a los usuarios y para dar el correcto servicio en sus unidades. Pero, a la vez, para estar suficientemente capacitados antes de que el futuro llegue a las tareas que realizan desde un presente ya inestable. Entrarían aquí en juego modalidades 
de enseñanza apropiadas para hacerlo, como la enseñanza a distancia para la actualización técnica y el aprendizaje permanente de los profesionales que viven en las grandes ciudades y de quienes lo hacen lejos de los campus (BOLAÑOS et al., 2013). Incluso habría que ofrecer una formación universitaria también híbrida y transdisciplinar de acuerdo con la transformación, complejidad y especialización laborales que les concediesen las competencias y destrezas que necesitan en su desempeño.

\section{CONCLUSIONES}

Las TIC han reformulado nuestra relación con los contenidos documentales desde su creación, así como en su comunicación, acceso y disponibilidad. La virtualidad de los procesos y objetos ha desdibujado la realidad material de los documentos y de su tratamiento, arrastrando con ello unos cambios profundos en las tareas que los profesionales ejecutan, en sus lugares, organización y métodos de trabajo y hasta en sus perfiles de actuación. Con inevitables consecuencias en la formación y en las capacidades que se esperan en su actuación diaria. La transformación digital ha construido una profesión sobre unas bases distintas que reflejan la manera en que representamos y comprendemos la vida. $Y$ con unos perfiles nuevos que no cuentan con suficientes profesionales para cubrir la demanda del mercado de trabajo

Todo lo que hasta ahora era manual en las tareas rutinarias primero se automatizó, luego se enlazó. De forma que las tareas profesionales se realizan en equipos participativos. Se demuestra que la evolución de nuestro sector depende de unas TIC que llevan treinta años siendo el futuro. Hace tiempo que se olvidó almacenar en cajas o por volúmenes y elaborar o comprobar registros, pues cualquier tarea ha pasado a hacerse de manera colaborativa y enlazada. En especial ha incidido en la adecuada distribución de los objetos de información: análisis de datos para conocer lo que quieren los usuarios, márketing digital para ofrecerles servicios o aplicaciones informáticas, gestión digital sobre los productos y procesos informativos.

Se van difuminando los perfiles profesionales relacionados con los espacios físicos de las bibliotecas, archivos y servicios de documentación. De manera que desde la estabilidad de tareas y puestos de trabajo se ha pasado a una complejidad profesional donde los límites de las titulaciones de origen se desdibujan ante problemas cuyo estudio y aplicación hay que abordar desde enfoques compuestos para apreciar los espacios comunes y con objeto de producir nuevas soluciones. La complejidad del territorio ilimitado que recorren los profesionales de la información no se puede dividir en pedazos ni envolverse en garantías integrales, solo queda atravesarlo en grupos colaborativos. 
No debe de extrañarnos esta situación, pues la naturaleza de la información y sus aplicaciones son transdisciplinares. Más desde que la tecnología electrónica ha unificado el formato de los recursos.

Las TIC son el principal factor de cambio en la manera de actuar los servicios de información. A medida que aparecen sucesivas modificaciones, los profesionales perciben la necesidad de mejorar y adaptar sus habilidades a lo que esperan sus organizaciones. Volviéndose permanentes las dudas sobre su adecuado nivel de capacitación. Estas dificultades se relacionan con el desafío de organizar el trabajo para los previsibles movimientos inmediatos. Lo que fuerza a los gerentes de las unidades y sistemas a intuir las tendencias y las competencias indispensables no solo para cuidar las necesidades actuales de los usuarios, sino para anticiparse a las futuras. A la hora de adecuarse a unos perfiles y ocupaciones transdisciplinares para atender al desafío de trabajar de otra forma, en tareas renovadas, la adquisición de nuevas habilidades o la actualización de las que ya se detentan pide prolongar y ampliar la formación, lo que se antoja irrealizable cuando los presupuestos se reducen.

Estamos inmersos en una coyuntura progresiva en la que nuestras profesiones muestran un carácter híbrido, no pueden quedarse cerradas en lo documental. Los profesionales de la información necesitan incorporar nuevas competencias y habilidades ante la evolución del sector y de las disciplinas que lo fundamentan. Y, desde luego, ante las exigencias del mercado de trabajo. Resulta muy favorable para esas nuevas competencias que sean transferibles a múltiples sectores. No es necesario que se desarrollen en unidades y servicios de información. De modo que se han abierto nuevas posibilidades de inserción laboral en cualquier sector con presencia digital en todo tipo de empresas y administraciones públicas, además del sector de la información.

El mercado laboral solicita profesionales capacitados para manejar aplicaciones y recursos digitales, que estén aptos para planificar los servicios adecuados y para gestionar el conocimiento en empresas y entidades. También se valora la idoneidad para jugar el papel de auditores y consultores dentro de sus organizaciones e instituciones. En la última década ha sido notorio el crecimiento en la demanda de empleo para esos profesionales, según lo revelado en los estudios analizados.

Trabajan, genéricamente, dentro de estructuras organizativas transdisciplinares que disponen de buenos medios tecnológicos para crear, tratar y gestionar información digital. Con el empleo de plataformas digitales, su actividad se dispersa por áreas como la comunicación académica; los archivos digitales; la industria de los contenidos; los recursos educativos, principalmente abiertos; la custodia de datos, en especial de investigación; la gestión de proyectos; las humanidades digitales; las soluciones de información personalizadas; la visualización y los objetos digitales. 


\section{REFERENCIAS}

ALA (2016). The State of America's Libraries 2017: A Report from the ALA. Kathy S. Rosa, ed. Disponible: www.ala.org/news/state-americas-libraries-report-2017

BAHIA JACINTHO, E. El mercado de trabajo para archiveros según los anuncios brasileños de empleo (2012-2014): análisis y organización terminológicos de ofertas empresariales. Tesis doctoral. Getafe: Universidad Carlos III de Madrid, 2016.

BAHIA JACINTHO, E.; MOREIRO-GONZÁLEZ, J. A. Archiveros y documentalistas emprendedores: Ios casos español y brasileño. Informação \& Sociedade: Estudos, v. 24, n. 2, 2014, p. 121-138.

BERNERS-LEE, T., HALL, W., HENDLER, J., SHADBOLT, N., \& WEITZNER, D. J. Creating a Science of the Web. Science, 313, n. 5788, 2006.

BOLAÑOS-MEJÍAS, C., BUENO DE LA FUENTE, G., ALVES, F. M. 0 protagonismo da informaçãodocumentação na cooperação ao desenvolvimento: os recursos educativos abertos nos processos de e-learning. Informação \& Sociedade: Estudos, v. 23, n. 1, 2013.

BRYNJOLFSSON, E.; MCAFEE, A. Race against the machine. Lexington: Digital Frontier, 2011.

CASTELLS, M. The new public sphere: Global civil society, communication networks, and global governance. The annals of the American Academy of Political and Social Science, v. 616, n. 1, 2008.

CHAWNER, B.; OLIVER, G. What if? Exploring alternative models for professional LIS education. The Australian Library Journal, v. 65, n. 4, 2016.

CHOI, Y., RASMUSSEN, E. What qualifications and skills are important for digital librarian positions in academic libraries? A job advertisement analysis. The journal of academic librarianship, v. 35, n. 5, 2009.

DAVID, H. Wiring the labor market. Journal of Economic Perspectives, v. 15, $n^{0}$ 1, p. 25-40, 2001.

DE LIMA, R.C.M. Estratégias de informação e modelos organizacionais: o espaço da administração e da comunicação na ciência da informação. Rio de Janeiro: Editora E-papers, 2007.

ESPAÑA. Benchmarking de la situación de los estudios correspondientes o afines en Europa y el resto del Mundo. En Libro Blanco para el Diseño de Titulaciones Universitarias en el Marco de la Economía Digital. Madrid: Ministerio de Industria, Energía y Turismo. p. 145-160, 2015.

EUROPEAN COMMISSION. Interactive content and convergence: Implications for theinformation society. London: DG Information Society and Media, 2006. http://www.rcc.gov.pt/ SiteCollectionDocuments/interactive_content_ec2006.pdf

FTI-AMETIC. Estudio de Perfiles Profesionales más demandados en el ámbito de los Contenidos Digitales en España 2012-2017. Profesionales TIC. Madrid: Fundación de Tecnologías de la Información-AMETIC, 2012.

INESDI. Vademécum de las profesiones digitales 2015. Barcelona: INESDI Digital Business School, 2015.

LEIVA-AGUILERA, J. Comunicación en la empresa y apertura del perfil profesional de los documentalistas. El profesional de la información, v. 19, n. 2, p. 117-121, 2010. 
LYON, L. Transparency: the emerging third dimension of Open Science and Open Data. Liber quarterly, v. 25, no 4. pp. 153-171, 2016.

MODESTO, F. Panorama da catalogação no Brasil: da década de 1930 aos primeiros anos do século XXI. En, XXII CBBD. Congresso Brasileiro de Biblioteconomia, Documentação e Ciência da Informação. Brasília (DF), 08 a 11 de Julho de 2007. p. 1-22, 2007.

MOREIRO-GONZÁLEZ, J. A., SÁNCHEZ-CUADRADO, S., MORATO-LARA, J. L., TEJADA- ARTIGAS, C. M. Creación de un corpus coordinado de competencias en información y documentación a partir de ofertas de empleo en España. RISTI: Revista Ibérica de Sistemas e Tecnologias de Informação, nº 3, p.79-91, 2009.

MOREIRO, J. A. Introducción Bibliográfica y Conceptual al Estudio evolutivo de la Documentación. Barcelona: P.P.U, 1991.

ORTIZ-REPISO, V.; CALZADA-PRADO, J.; APORTELA-RODRÍGUEZ, I. M. ¿Qué está pasando con los estudios universitarios de biblioteconomía y documentación en España? El profesional de la información, v. 22, $n^{0}$ 6, p. 505-514., 2013

PARK, J., LU, C., MARION, L. Cataloging professionals in the digital environment: A content analysis of job descriptions. Journal of the Association for Information Science and Technology, v. 60, $n^{\circ} 4$, p. 844-857, 2009.

REYES, A., BARRETO, C., CERDEIRINHA, J., de SÁ GUEDES, M., TEIXEIRA, P., NÉO, S. Gestor e curador da informação: tendências, perfis e estratégias de reconhecimento. Páginas a\&b: arquivos e bibliotecas, p. 3-15, 2017.

ROSS, L.; SENNYEY, P. The library is dead, long live the library! The practice of academic librarianship and the digital revolution. The Journal of Academic Librarianship, v. 34, n. 2, p. 145-152, 2008.

RUBIN, R. E., JANES, J. Foundations of library and information science. 4th ed. New York-London: ALA\&Neal-Schuman, 2017.

SAYÃO, L.F., SALES, L.F. Curadoria digital: um novo patamar para preservação de dados digitais de pesquisa. Informação\&Sociedade: Estudos.v.22, n. 3, p.179-191, 2012.

SHANON, T.; WEAVER, W. Teoría matemática de la comunicación. Madrid: Forja, 1981.

TAM, L.; ROBERTSON, A. C. Managing change: libraries and information services in the digital age. Library Management, v. 23, n 8-9, p. 369-377, 2002.

TAPSCOTT, D. The digital economy: Promise and peril in the age of networked intelligence. New York: McGraw-Hill, 1996.

TEJADA-ARTIGAS, C.; CHACÓN JARÉN, S.; MOREIRO-GONZÁLEZ, J. A. (2014). Mercado de trabajo en información y documentación y crisis económica en España: una aproximación a partir de las ofertas publicadas en IweTel entre 2008 y 2013. BiD: Textos universitaris de biblioteconomia i documentació, v. 32, n. 32. Disponible: http://bid.ub.edu/es/32/tejada2.htm.

VERGUEIRO, W.; MOREIRO-GONZÁLEZ, J. A. Towards a new information-documentation professional: competencies and attitudes required for the Brazilian labor market. In, ALMEIDA, F.A.S.; Malheiro, A. S.; Franco, M.J.B.; Freitas, C.C. (Org.). Information management. Selected papers from Coletânea LusoBrasileira. Porto: Faculdade de Letras. Universidade do Porto. v. 6, p. 151-164, 2015.

WOLTON, D. Internet ¿y después? Barcelona: Gedisa. 